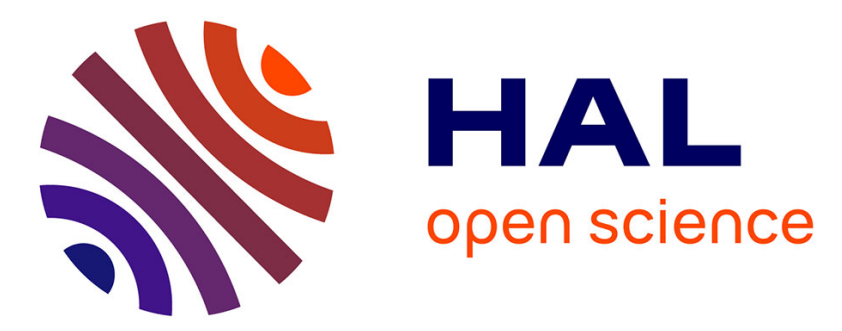

\title{
An evidential k-nearest neighbors combination rule for tree species recognition
}

\author{
Siwar Jendoubi, Didier Coquin, Reda Boukezzoula
}

\section{To cite this version:}

Siwar Jendoubi, Didier Coquin, Reda Boukezzoula. An evidential k-nearest neighbors combination rule for tree species recognition. 5th International Conference, Belief 2018, Sep 2018, Compiègne, France. hal-01953169

\author{
HAL Id: hal-01953169 \\ https://hal.science/hal-01953169
}

Submitted on 12 Dec 2018

HAL is a multi-disciplinary open access archive for the deposit and dissemination of scientific research documents, whether they are published or not. The documents may come from teaching and research institutions in France or abroad, or from public or private research centers.
L'archive ouverte pluridisciplinaire HAL, est destinée au dépôt et à la diffusion de documents scientifiques de niveau recherche, publiés ou non, émanant des établissements d'enseignement et de recherche français ou étrangers, des laboratoires publics ou privés. 


\title{
An evidential $k$-nearest neighbors combination rule for tree species recognition
}

\author{
Siwar Jendoubi ${ }^{1,2}$, Didier Coquin ${ }^{1}$ and Reda Boukezzoula ${ }^{1}$ \\ 1 LISTIC, University Savoie Mont Blanc, 74944 Annecy-le-Vieux, France \\ 2 LARODEC, University of Tunis, ISG Tunis, 2000 Le Bardo, Tunisia \\ \{siwar.jendoubi, didier.coquin, reda.boukezzoula\}@univ-smb.fr
}

\begin{abstract}
The task of tree species recognition is to recognize the tree species using photos of their leaves and barks. In this paper, we propose an evidential $k$-nearest neighbors $(k-\mathrm{NN})$ combination rule. The proposed rule is adapted to classification problems where we have a large number of classes with an intra-class variability and an inter-class similarity like the problem of tree species recognition. Finally, we compare the performance of the proposed solution to the evidential $k$-NN.
\end{abstract}

Keywords: Tree species recognition, Belief functions theory, $k$-NN.

\section{Introduction}

The tree species recognition ${ }^{3}$ searches to identify the tree species through photos of leaves and barks taken with a smartphone. The automation of this task is very useful for non botanist users who want to learn more about trees. The idea is to help a user and to teach him how to recognize tree species. Trees recognition is a challenging classification problem.

The $k$-nearest neighbors $(k-\mathrm{NN})$ classification is a fundamental and simple technique. In fact, all we need to use it is a training set that contains a representative labeled data sample of all possible classes in a given problem and a distance metric. Then, to classify a new data point $x, k$-NN computes the point distance with all points in the training set and selects the $k$-nearest neighbors. Finally, $k$-NN chooses the class of $x$ according to the majority vote principle. The $k$-NN classifier is sensitive to the value of its main parameter $k$.

Denœux [6] introduced a $k$-NN decision rule based on the theory of belief functions [9]. The advantage of this rule is that it considers the distances from the nearest neighbors in the decision step (classification step) which leads to more accurate results. In fact, Denœux's solution combines the evidence from the nearest neighbors through the framework of the theory of belief functions. The resulting decision rule is more robust to the conflicting information, i.e. when the object to be classified is close to different classes, and information

\footnotetext{
${ }^{3}$ This work has been supported by the French National Agency for Research with the reference ANR-15-CES38-0004 (ReVeRIES project).
} 
(a)

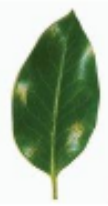

(b)

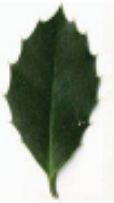

(c)

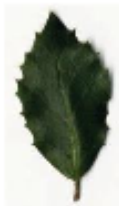

Fig. 1. Intra-species variability and interspecies similarity: (a) and (b) belong to the holly species and (c) belongs to the oak species

sparsity, i.e. when the object to be classified is far from all patterns in the training set.

The evidential $k$-NN (Ek-NN) is accurate in many existing classification problems. However, when we have a large number of classes, this accuracy may decrease. This problem becomes serious when we have an intra-class variability and an inter-class similarity like the problem of tree species recognition. This fact increases the conflict between species and leads to miss classification. This limitation was addressed in the literature. In fact, [11] and [8] proposed variants of the E $k$-NN based on the Parametric Conjunctive t-Rules and an hybrid Dempster-Yager Rule respectively.

The main contributions of this paper are the following: first, we propose an evidential $k$-NN rule that is more adapted for the large number of classes problem. Besides, the proposed solution deals with the conflict between species and reduces it. In fact, we propose to use a modified version of the large number of source combination algorithm introduced by Zhou et al. [13]. Second, we prove the performance of the proposed solution through a set of experiments on the trees recognition problem. Then, we show that the proposed solution is more accurate than the $\mathrm{E} k$-NN.

This paper is organized as follows: Section 2 is dedicated to present the tree species recognition problem. Section 3 details the E $k$-NN trees recognition system. Section 4 introduces the combination rule for large number of classes recognition. Section 5 presents some experiments. Finally, Section 6 concludes the paper.

\section{Tree species recognition}

The trees species recognition is the problem of identifying trees from their leaves, barks, flowers, etc. In this work, we are interested in recognizing trees from their leaves and barks. In the nature, recognizing trees is not an easy task and it needs a botanist. In these last years, many researches were conducted to automate this task. However, this is not a simple task. Indeed, in the nature there is a large variety of species. Besides, it is very common to find similarities between different species and a variability of trees in the same species. Let take the example of leaves in Figure 1, the leaves (a) and (b) look different, but they belong to the holly species. Besides, the leaves (b) and (c) are very similar, but they belong to two different species (holly and oak). 
In the literature, many solutions was proposed for this problem [7,3,1]. Besides, many smartphone applications are now available like Pl@ntNet and Folia. $\mathrm{Pl} @$ ntNet [7] gives good recognition rates. However, it needs to be connected to Internet. Moreover, it provides the results without any explanation. Whereas, we want to provide the user with information to help him get in the world of botany. In the other hand, we have Folia [3], this application does not need Internet connection. In fact, it recognizes the tree through a limited number of attributes extracted from leaves photos then it can be run on a smartphone. Besides, this application explains to the user its results. However, the current version of Folia recognizes only photos of leaves.

Ben Ameur et. al. [1] introduced a model-based evidential solution that recognizes trees from leaves and barks. Their solution classifies the leaf and bark photos of the tree using the random forest classifier. Then, they use the inverse pignistic [12] operator to transform the classification results to a consonant mass distribution. Next, they fuse the consonant masses of leaf and bark to obtain a mass distribution that is used to recognize the tree. In this paper, we search to improve the results of [1]. Furthermore, we want to avoid the random forest classification step that is consuming in execution time.

In the next section, we detail the evidential $k$-NN species recognition system.

\section{Evidential $k$-nearest neighbors for trees recognition}

To recognize tree species from leaves and barks, we follow the botanists strategy. In fact, they identify the different morphological characteristics of leaves (apex, shape, etc) and barks (color, gabor, etc). Then, we extract from each characteristic a vector of attributes to characterize it. From each leaf photo, three vectors are extracted. The first one characterizes the apex and the base of the leaf, the second represents the margin and the last characterizes the polygonal model. From each bark photo, four attributes vectors are extracted which characterize respectively: 1) the color hue $\mathrm{H}$ of the HSV space, 2) the texture (gabor) space, $3 \& 4)$ vertical and horizontal orientation of the bark texture. Bertrand et al. [2] detail these characteristics. We consider each characteristic as a source of information.

We define a classification system as described in Figure 2.This architecture is useful to provide the user with classification results according to each characteristic separately and with an explanation of the results. Next, to classify a new leaf/bark photo, we extract the same characteristics. Then, we apply an evidential $k$-NN (Ek-NN) on each characteristics as presented in Figure 2. The output of each $\mathrm{E} k$-NN is a BBA distribution defined on the frame of all possible species. Next, we combine the obtained BBAs to make a decision according to leaves, barks and combined leaves and barks.

The E $k$-NN [6] starts like the probabilistic $k$-NN by estimating the distance between an unclassified object $x$ and all the elements in the training set. Next, it takes the $k$ neighbors having the least distances to $x$. At this step, the probabilistic $k$-NN chooses the class of $x$ according to the majority vote principle. 


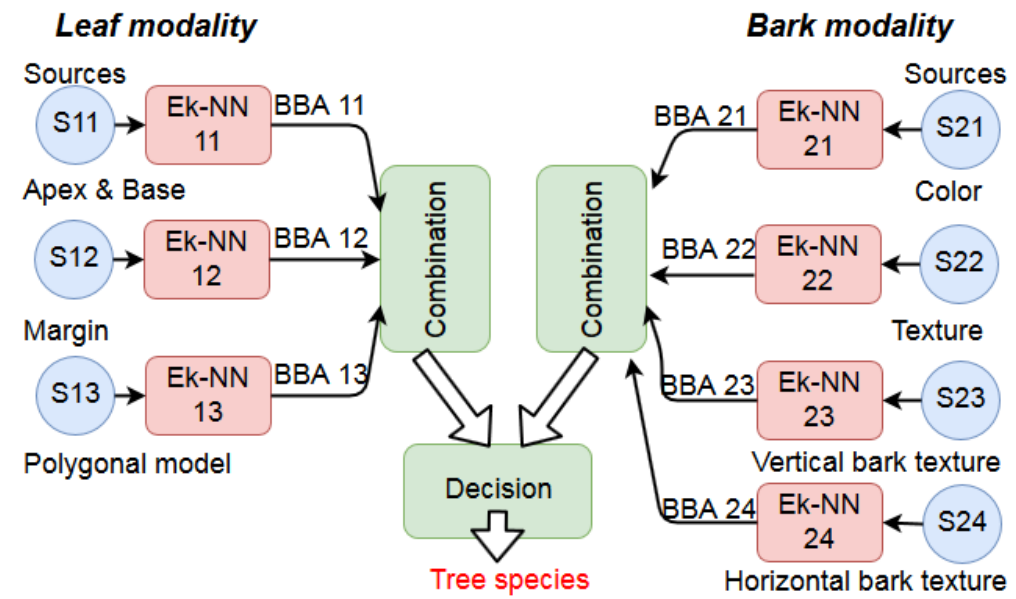

Fig. 2. Evidential $k$-NN based species recognition

Whereas, E $k$-NN estimates a mass distribution for each nearest neighbor, $i$, using its distance to $x$. Let us define the frame of discernment $\Omega=\left\{c_{1}, c_{2}, \ldots, c_{n}\right\}$. The $\mathrm{E} k$-NN estimates the mass $m_{i}^{\Omega}$ for each nearest neighbor $i$ as follows:

$$
\begin{aligned}
& m_{i}^{\Omega}\left(c_{i}\right)=\alpha_{0} \Phi_{i}\left(d_{i}\right) \\
& m_{i}^{\Omega}(\Omega)=1-m^{\Omega}\left(c_{i}\right)
\end{aligned}
$$

such that $c_{i}$ is the class of the neighbor $i, d_{i}$ is the distance between $x$ and $i$ and $\Phi_{i}$ is a decreasing function that may be defined as $\Phi_{i}\left(d_{i}\right)=e^{-\gamma_{i} d_{i}^{\beta}}$ where $\gamma_{i}>0$ and $\beta \in[0,1]$. Then, the $\mathrm{E} k$-NN combines the obtained mass functions from all the neighbors in order to obtain a decision mass distribution. The combination is done, generally, using the Dempster's rule [5]. Finally, the decision may be taken using the maximum pignistic.

The inter-species similarities and the intra-species variability make harder the recognition task for the Ek-NN. These two facts lead to an imprecise and uncertain environment for the $\mathrm{E} k$-NN. Besides, they generates an important conflict between the species. Given the example presented in Figure 3, we have a leaf and we want to identify its species. The Ek-NN selects the $k$ nearest neighbors to the given leaf. In Figure 3 we have many species that are near to the unknown leaf. If we take a small $k$ value ( $k=3$ for example), the true class (C6 in this case) will not appear in the nearest neighbors set (C1, C4, C5). Then, we need to choose a big value of $k$ in order to increase the probability that the true class appears. In our experiments we fixed $k$ to 20 according to an Akaike's information criterion (AIC) [4].

After selecting the nearest neighbors, the $\mathrm{E} k$-NN estimates a simple BBA for each selected neighbor. Besides, the distances between the unknown leaf and its nearest neighbors are small and almost equal. Then we will obtain $k$ BBA with a high amount of belief on the neighbor's class $\left(C_{i}\right)$. Combining these BBAs leads 


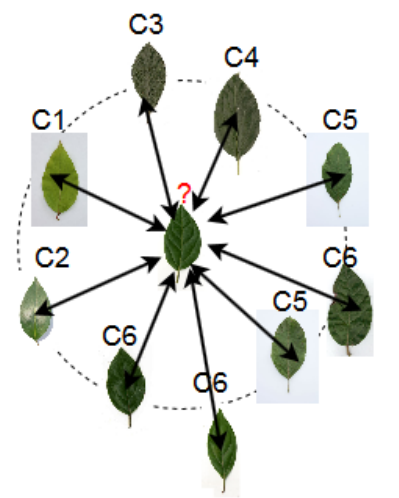

C1: cornus mas I

C2: ligustrum vulgare I

C3: prunus spinosa I

C4: rhamnus cathartica I

C5: sorbus aria crantz

C6: vuburnum tinus I

Fig. 3. Inter-species similarities and intra-species variabilities effects on the selected nearest neighbors

to a high conflict value (mass of the empty set $m(\emptyset)$ ) that tends to 1 . This fact leads to a miss classification even when we use the Dempster's rule.

\section{Proposed evidential $k$-nearest neighbors combination rule}

To better distribute the conflict and to resolve the combination problem introduced in the previous section, we propose to combine the BBAs estimated from the nearest neighbors using the large number of sources combination algorithm (LNS) proposed in [13]. LNS takes as input a set of simple masses, i.e. a mass with two focal elements among them $\Omega$. Then, the LNS input is the set of mass functions estimated from neighbors. In the first step, LNS clusters the masses into $\theta$ clusters according to their focal element $A \neq \Omega$. Next, it combines each cluster masses using a combination rule ([13] used the conjunctive rule of combination (CRC) [10]).

After combining the cluster masses, we obtain $\theta$ masses, one by cluster. The next step is the reliability-based discounting step. Then, the clusters are seen as sources of information and the main purpose is to consider the reliability of each cluster through the following hypothesis: the larger the number of masses in the cluster $\theta_{j}$ is, the more reliable the cluster $\theta_{j}$ is. Then, we propose to estimate the reliability coefficient of the $j^{\text {th }}$ cluster as $\varepsilon_{j}=\frac{\operatorname{Card}\left(\theta_{j}\right)}{k}$. Next, each cluster mass is discounted using its reliability coefficient. The last step of the LNS algorithm is to combine all discounted cluster masses using a combination rule ([13] used the $\mathrm{CRC}$ ).

The advantage of the LNS algorithm is that it offers the possibility to use two different combination rules as it combines masses in two levels, i.e. intra and inter-clusters. Then, we propose the disjunctive rule of combination (DRC) [10] to combine intra-cluster masses and the CRC to combine the inter clusters masses. In the next section, we present a set of experiments on the tree 


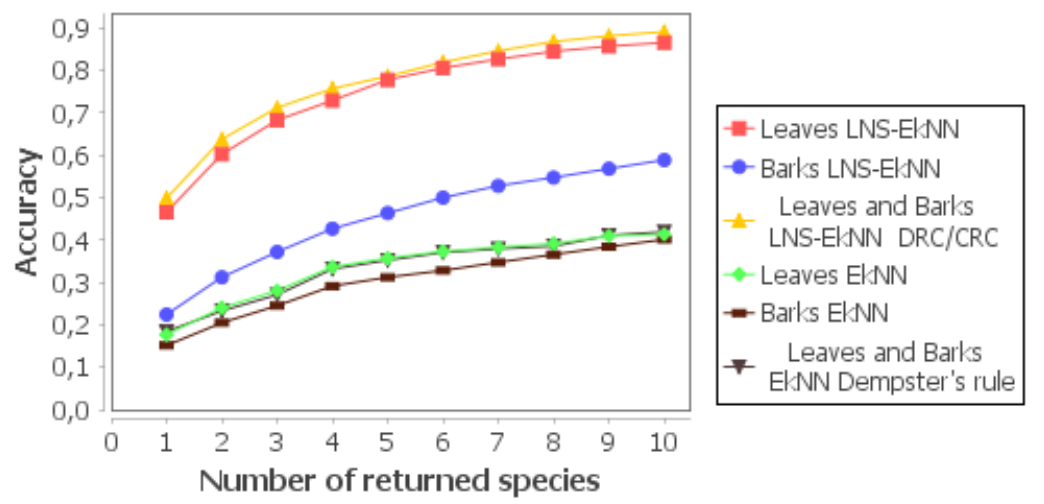

Fig. 4. Comparison between the proposed LNS evidential $k$-NN and the Denœux's evidential $k$-NN according to leaves, barks and combined leaves and barks accuracy

species recognition problem and we show the contribution of the DRC in the LNS algorithm.

\section{Experiments}

To evaluate the proposed solution, we use a dataset from the ImageClef challenge. The dataset contains photos of trees from mainland France. Those photos are taken in the wild by non-professionals. Then, the photos in this dataset are similar to those a user may take in the nature. The dataset contains 2572 leaves photos and 895 barks photos for training and 820 couple of leaves and barks photos for testing. In the dataset, we have 72 tree species to recognize. In the experiments, we fixed the $k$ parameter to 20 according to an AIC criterion, $\alpha_{0}=0.944, \gamma_{i}=0.76$ and $\beta=3.6$. We note that the classifier returns the ten most likely tree species. Then we compare LNS evidential $k$-NN rule (LNS E $k$ $\mathrm{NN}$ ) with the $\mathrm{E} k$-NN according to the accuracy that the good species is among the first ten returned species.

In a first experiment, we compare the proposed LNS E $k$-NN with the E $k$-NN using the Dempster's rule to combine the $k$ masses. We used the conjunctive combination rule to combine the classification results of leaves characteristics and those of barks, and we used the disjunctive combination rule to combine the results of leaves and barks. Figure (4) presents the classification results of the two experimented classifiers according to leaves characteristics, barks characteristics and combined leaves and barks. According to Figure (4), the Ek-NN is not efficient as it gives low classification rates. Whereas, the proposed solution gives good classification rates. In fact, we have got an accuracy equals to $49.87 \%$ for the first recognized species and $89.14 \%$ for ten species from the combined leaves and barks classifiers.

In a second experiment, we study the impact of the combination rule on the accuracy of the combined leaves and barks classifiers. Results of this experiment 


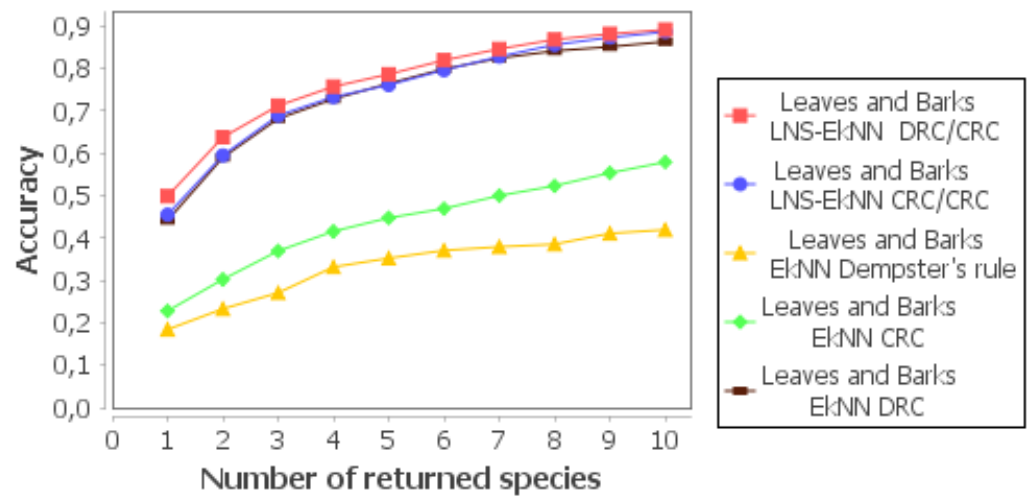

Fig. 5. Impact of the combination rule on the classifier accuracy: fusion of leaves and barks classifiers

are shown in Figure (5). Then, we tested the Ek-NN with the Dempster's rule, CRC and DRC. We notice that the used combination rule has an important impact on the accuracy of this classifier. In fact, the CRC and the DRC succeeded to improve the accuracy of the E $k$-NN.

We said in the previous section that the LNS algorithm allows the use of two different combination rules to combine intra and inter-clusters masses. Then in Figure (5), we present the accuracy using the DRC to combine intra-cluster masses and the CRC to combine the inter clusters masses compared to the accuracy when we use the CRC for both of them as proposed by [13]. According to this experiment, the DRC has a positive impact on the accuracy of the proposed LNS evidential $k$-NN classifier. Then, we have $49.87 \%$ for the first species recognized with the DRC (red curve) against $45.48 \%$ when we use only the CRC (blue curve). Besides, we have $89.14 \%$ for ten species with the DRC (red curve) against $88.56 \%$ when we use only the CRC (blue curve). Furthermore, the effect of the DRC is more important for the first eight detected species. In fact, when we use the DRC combination rule to combine intra-clusters masses, the mass value on the global ignorance, i.e. $\Omega$, in the resulting distribution is more important than its value when we use the CRC, and this fact, allowed the classifier to more consider the uncertainty and to improve the results as shown in Figure (5).

From the experiments, we can conclude that the LNS combination algorithm is adapted to combine the estimated masses of the evidential $k$-NN. In fact, as we see above the proposed LNS evidential $k$-NN classifier is more accurate in recognizing tree species than the $\mathrm{E} k-\mathrm{NN}$.

\section{Conclusion}

To sum up, in this paper we focus on the problem of tree species recognition which is a challenging task. We propose the LNS evidential $k$-nearest neighbors 
classifier. This solution is more adapted for classification when we have a large number of classes problem. In fact, the LNS combination algorithm is permanent to combine evidence from large number of sources [13] and to deal with the conflict which is the case of the problem in this paper.

In the future works, we will search to improve the achieved results in order to give more accurate recognition to the end user. Then, we will search to optimize the E $k$-NN parameters according to [14]. Another good solution may be to use an adapted classifier for each extracted characteristic from leaves and barks.

\section{References}

1. Ben Ameur, R., Coquin, D., Valet, L.: Influence of the basic belief assignments construction on the behavior of a fusion system for tree species recognition. In: Proceedings of 20th International Conference on Information Fusion, Xi'an, China, IEEE FUSION (July 2017)

2. Bertrand, S., Ameur, R.B., Cerutti, G., Coquin, D., Valet, L., Tougne, L.: Bark and leaf fusion systems to improve automatic tree species recognition. Ecological Informatics 46, 57-73 (June 2018)

3. Cerutti, G., Tougne, L., Mille, J., Vacavant, A., Coquin, D.: Understanding leaves in natural images-a model-based approach for tree species identification. Computer Vision and Image Understanding 117(10), 1482-1501 (2013)

4. Cetin, M.C., Erar, A.: Variable selection with akaike information criteria: a comparative study. Hacettepe Journal of Mathematics and Statistics 31, 89-97 (2002)

5. Dempster, A.P.: Upper and Lower probabilities induced by a multivalued mapping. Annals of Mathematical Statistics 38, 325-339 (1967)

6. Denœux, T.: A $k$-Nearest Neighbor Classification Rule Based on Dempster-Shafer Theory. IEEE Transactions on Systems, Man, and Cybernetics - Part A: Systems and Humans 25(5), 804-813 (May 1995)

7. Goëau, H., Bonnet, P., Joly, A., Bakić, V., Barbe, J., Yahiaoui, I., Selmi, S., Carré, J., Barthélémy, D., Boujemaa, N., Molino, J.F., Duché, G., Péronnet, A.: Pl@ntnet mobile app. In: Proceedings of the 21st ACM Int. Conf. on Multimedia. pp. 423-424 (2013)

8. Lian, C., Ruan, S., Denœux, T.: An evidential classifier based on feature selection and two-step classification strategy. Pattern Recognition 48(7), 2318-2327 (July 2015)

9. Shafer, G.: A mathematical theory of evidence. Princeton University Press (1976)

10. Smets, P.: Belief Functions: the Disjunctive Rule of Combination and the Generalized Bayesian Theorem. Int. J. of Approximate Reasoning 9, 1-35 (1993)

11. Su, Z.G., Denoeux, T., Hao, Y.S., Zhao, M.: Evidential k-NN classification with enhanced performance via optimizing a class of parametric conjunctive t-rules. Knowledge-Based Systems 142, 7-16 (February 2018)

12. Sudano, J.J.: Inverse pignistic probability transforms. In: Proceedings of FUSION. pp. $763-768$ (2002)

13. Zhou, K., Martin, A., Pan, Q.: Evidence combination for a large number of sources. In: Proceedings of 20th International Conference on Information Fusion, Xi'an, China, IEEE FUSION (July 2017)

14. Zouhal, L.M., Denœux, T.: An Evidence-Theoric $k$-NN Rule with Parameter Optimization. IEEE Transactions on Systems, Man, and Cybernetics - Part C: Applications and Reviews 28(2), 263-271 (May 1998) 